\title{
The Operation Recommendation based on QoS in Service Substitution Environment
}

\author{
Yun-Young Hwang ${ }^{1}$, Jungsun Yoon ${ }^{1}$ and Kyu-Chul Lee ${ }^{2, *}$ \\ ${ }^{1}$ Korea Institute of Science and Technology Information, Korea \\ ${ }^{2}$ Chungnam National University, Korea \\ ${ }^{1}$ \{yyhwang, jsyoon\}@kisti.re.kr \\ ${ }^{2} k c l e e @ c n u . a c . k r$

\begin{abstract}
\end{abstract}
In ubiquitous computing environments, users usually compose several services to achieve their goals. The service composition method of this paper is based on WSUN (Web Services on Universal Networks) [7]. It supports dynamic sevice composition which takes account of the dynamic factors of ubiquitous environments. In service composition, an operation of a service should be substituted by another operation when the existing operation is not available. The substitutable operation should support same functionality of existing operation. Moreover, it should be able focommunicale with operation back and forth. The service composition mechanism of WSUN cansiders the functional similarity between unavailable operations and substitutable operation but it does not take account of input and output message structures and parameter types of operations. In order to enhance WSUN service substitution method, we use SAWSDL (Semantic Annotation WSDL for XML Schema) [15], which is Semantic Web Services technique. SAWSDL provides two construct: modelReference and SchemaMapping. A modelReference specifies the association between a WSDL or XML SChema component and a concept in some semantic data. Semantic annotations using nodelReference help to discover substitutable operations. In addition, a schemaMapping solves the Reterogeneity of I/O message structures and parameter types of operations. Consequently, even if the operation, which has same functionality of unavailable operation, has different I/O message structures and parameter types of unavailable operation. In order to recommend the operation has good quality, we define the quality of operation. It is a criveria that user can choose the best operation. In addition, we take a user weight for acoommodation of user's requirements. The recommendation system prioritizes operation through calculating quality of operation based on user weight value. Therefore, operation by operation's priority recommends users. Our system supports substitutable operakion discovery mechanism using Semantic Web Services technique, and substitutable operations list to satisfy user's requirement recommend in ubiquitous environment. Therefore, user can discover substitutable operation in substitution situation. In addition, substitutable operations to fit user requirement recommend user according to weight value from user.

Keywords: service substitution, service recommendation, ubiquitous computing, QoS

* Corresponding Author 


\section{Introduction}

Recent Advances in computing and network technology, various services have increased exponentially. As a result, users can have a wide choice of services, and they choose the better quality of them among services, which have the same functionality. The arrival of ubiquitous computing environment, while the services are provided, devices including these services, and users can have a number of moving. Even under such environment, users want to provide 'seamless' service. To address this requirement, many researches used the method of service substitution. Substitution means replacing a component with another component, as long as the replacing component produces the same output and satisfies the same requirements as the replaced component [4]. In ubiquitous computing, we considered the following points. The fist, the user shall not be limited by the communication protocol. Each of the ubiquitous devices is conformed to different communication protocols. The second is frequently movement of devices and user. This paper proposed the operation recommendation approach providing 'seamless' service to user. The folloying scenario will help the understanding our approach.

Scenario: The music streaming service of smarphone'should be always used with sound output device such as speaker. If the user goes to company, he can continue to provide service with sound output device of the company. This device is lacated near the user, and it can communicate with his smartphone.

In order to realize this scenario, a list of alternative services is composited services, which have same functionality and are satisfied the user requirements. In this paper we propose an enhanced service substitution methoof service brokering approach (universal service broker: US-Broker) in [7]. The approach addresses interoperability and composition between heterogeneous ubiquitous services. The basic concept of US-Broker composition is to derive a mapping between the target operation that should be substituted and substitute operation that offers similar functionality through a different interface. In addition, [7] represents the operation grouping information grouped by operation functionality. If target operation and another operation ate belongs to same operation group, the operation is substitute operation. It is very simple, but it does not guarantee substitute service is executable because it does not consider I/O message structure and I/O parameter type. Furthermore, it does not take account of replacement costs or operation position.

To solve this problem, we suggest the method for service recommendation of semantic based service substitution in ubiquitous computing. Our approach is to enable the mapping between input of the one operation and output of the other operation. In addition, we can search the substrtute operation based on matching between I/O structure messages. Our approach can provide a list of ranking services considering replacement cost, degree of satisfaction of user requirements, availability, execution time of operation, and execution cost, and location of user and operation. To make ranking service list, we defined the weights to user requirement. The rest of the paper is structured as follows.

The major contributions of the paper are in defining and formalizing:

1) The equivalence relations between services considering the functionalities they propose via their functional interfaces. We define and formalize the service model and the service equivalence relations based on the semantic annotation of their interfaces and operations. These relations allow defining if two services are functionally equivalent or not.

2) Enhanced service substitution mechanisms for US-Broker executing in ubiquitous computing environments. Based on service equivalence relations, the ubiquitous computing environment can decide to substitute services by functionally equivalent ones. 
Next section introduces related work. Third section discusses our approach for substitution of stateful services. Finally, we present the conclusions of this study and further research related to this study.

\section{Related Work}

This section introduces the Web Services on Universal Networks (WSUN), and related work about service substitution. Our composition method is based on WSUN.

\subsection{Web Services on Universal Networks}

WSUN supports service brokering mechanism (US-Broker) for interoperability and dynamic composition of heterogeneous ubiquitous services [7]. US-Broker thanslates all ubiquitous services (such as Bluetooth services, ZigBee services, Jini services and etc.) to Web Services (called Virtual Web Services). This means service descriptions of all ubiquitous services are described in WSDL (Web Services Description Language) documents.

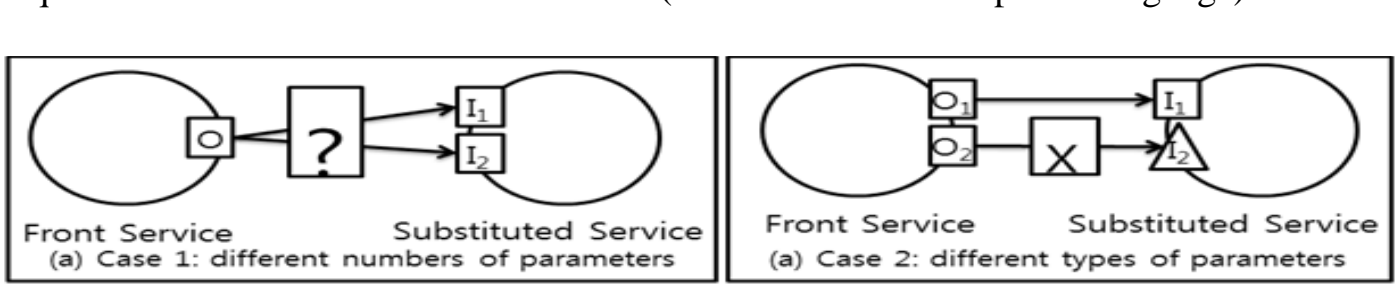

Figure 1. Consideting Cases cluring Substitution

US-Broker also supports simple mechanism for substitution of service. The basic idea is classifying services depends on their functionality. The services, which have same functionality, belong to same SerxiceGroup. One service can belong to one or more ServiceGroup, and ServiceGroup has also one or more services. If a service participating in combined services is not available or executable, US-Broker finds the substitutable services in same ServiceGroup with being substituted service. It is very simple and fast, but it does not guarantee substitute serrice is executable. The substituted service has to be able to communicate existing services are located at the front and the back of being substituted service.

We consider two cases to achieve these goals (Figure 1). The first case is the services, which are located the front and the back of being replaced service, and substituted service have different numbers of I/O parameters. The left case in Figure 1 shows this problem. The other case is there are inconsistent between two services (Figure 1 (b)). The both cases can appear between substituted service and front or back service of substituted service. USBroke cannot address two cases. Therefore, we enhanced US-Broker to cover these problems. To address these problems, we used the semantic technology is SAWSDL (Semantic Annotation of WSDL), because our service description is represented by WSDL standard. The next section described our detailed approach.

\subsection{Service Substitution Approach}

We represent two types of related work. The one is service substitution method, the other is related of quality of services.

Among the main challenges of [8], is the issue of service substitution for the application execution in such heterogeneous environments. In this article, we define a generic service model and describe the equivalence relations between services considering the functionalities 
they propose and their non-functional QoS properties. In order to determine the degree of similarity between two services, they compared between the concepts, operations, and interfaces. The similarity has four types: exact, plug-in, subsume, and fail. They measure the degree of similarity, after then discover the alternative service. However, this approach supports only the list of services, which have same I/O message structure and I/O parameter type.

[9] presents an approach whose objective is to support web services substitution. To perform Web services substitution with less impact on the ongoing, and sometimes critical, business processes, the approach proposes deploying communities of Web services. [11] uses the term "mapping rule" to express a single correspondence between the I/O parameters of operations to be adapted. However, if target service is not predefined into the mapping pale, it cannot support service substitution. It cannot consider the heterogeneous of I/Q types, either.

[11] defined the QoS such as capacity, availability, and security. The capacity includes throughput, responsiveness, information capacity, scalability, variability and consistency. Service availability considers availability, reliability, maintainability, resilience, variability, and constancy. Security has confidentiality, integrity, availability, authenticity, authority, and nonrepudiation. However, they assume that all levels of quality factors are given and did not propose a specific guideline to determine levels.

[12] defined the QoS for selection services are participated in the service composition. It considers execution price, execution durration, reputation, successful execution rate and availability.

[14] enhanced ISO/IEC9126 for ubiquitous computing. ISO/IEC9126 is standard about quality of services. [14] redefined ISO/IEC9126 in the light of the mobility. However, ISO/IEC9126 is mostly suitable for stand-alone applications, and has not been massively tested against web-based applications and ubiquitous computing applications. QoS-based operation composition has been the subject of various research efforts. Usually, the consumer's quality requirements are represented with quality properties. Then, mathematical or rule-based approaches are employed to find the best combination of operations for given quality constraints. 14. proposed a set of guidelines for service composition using user utility functions for QoS. However they did not present a mechanism for resolving conflictions between quality factors.

These approaches give only limited consideration to qualities such as response time and availability. In addition they evaluate quality by an objective function to be minimized (e.g., response time or cost) or maximized (e.g., availability) with constraints to be satisfied. To resolve conflictions between quality factors, we propose a quality-driven operation compositionmethodology for ubiquitous applications.

\section{Operation Recommendation Method}

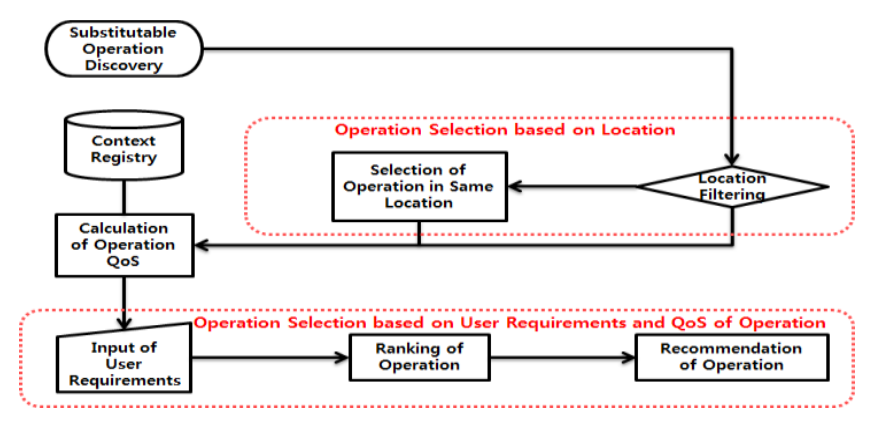

Figure 2. Operation Recommendation Process 
The following figure is shown an operation recommend process of our system. The first process is discovery of substitutable operation. The whether use or not of location filtering process is depending on positional relationship between the user and the operation. In the case of using location filtering process, user can choose the location in which they want to use the service. If the user determines the location, our system makes the target operation in the selection location. Conversely, if the user does not want to user location filtering process, the target operation is all operation through operation discovery process.

Next, an operation is selected to obtain the quality value, which value is stored in a registry context. Recommended candidate operations are stored in the registry operation context to derive the quality value might be used. For recommending operation to consider user requirements, our system requires the QoS weight from users. This weight is used as a tool for recommending operations.

The ranking operation process is composed into the following sub-processes. The first is formalization of quality of the operation, and next is T, Score calculation of quality of the operation. The final is the process of deriving the final value of quality of operation. Operation of the particular quality T-Score calculated to determine the ranking of the quality of the operation can be tailored to complement the problems. The final operations of quality values derived T-Score value of each operation input by the user by calculating a weighted user requirements required by the operation to the user is als 0 recommended as a high priority now for the process.

\subsection{Input/Output Mapping Approach}

Ubiquitous environment forms from the convergence of a variety of services and devices, and therefore, various quality factors are involved to provide transparent and seamless services. The substituted operation can communicate with the front and the rear operation. To facilitate this, we propose 1/9 mapping using SAWSDL (Semantic Annotation for WSDL). Figure 1 shows the example of heterógeneous I/O message structure between operation D and operation A, and heferogeneous $/ 0$ pgrameter type between operation $\mathrm{D}$ and operation $\mathrm{B}$. In order to operation $\mathrm{D}$ instead of operation $\mathrm{C}$, we need I/O mapping between heterogeneous I/O messages. We use SAWSDL component for I/O mapping. We define the ontology (operation group ontology), which describes operation functionality. Each I/O parameter refers ontology. The output message of operation A has same functionality as input message of operation B.

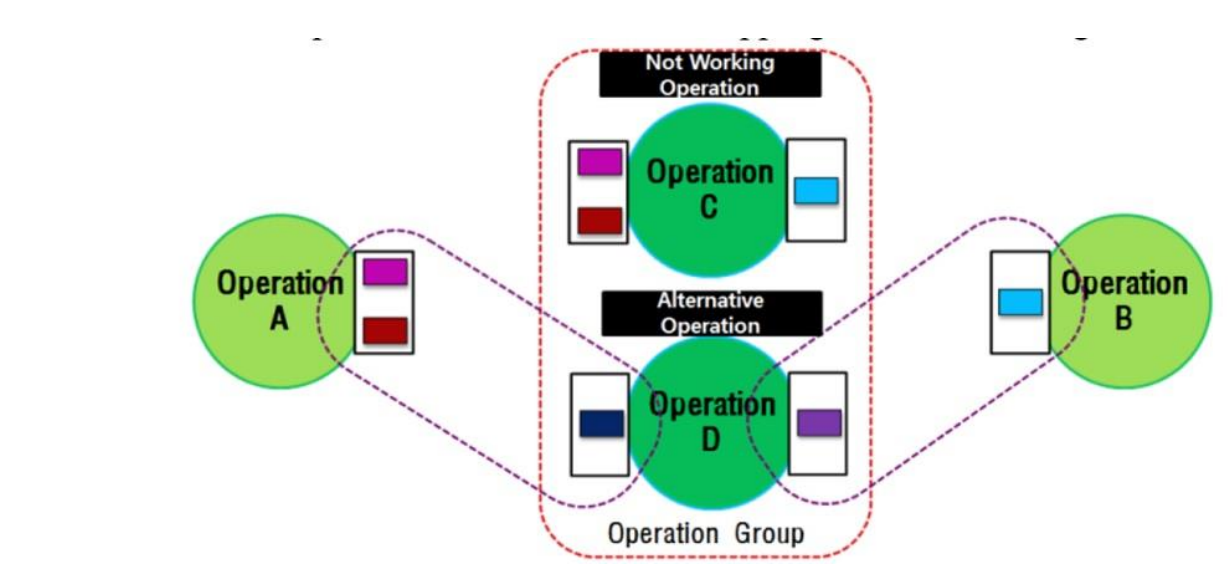

Figure 3. Example of Heterogeneous I/O Message Structure and I/O Parameter Type between Operations 
The process to find substitutable services consists of four steps. The first step is making first candidate services. US-Broker extracts services from ServiceGroup of target service should be substituted, and makes candidate substitutable services list. Next, US-Broker checks whether between the model reference of candidate service and existing service, which will connect to substituted service. This process is based on from Eq. 1 to Eq. 5.

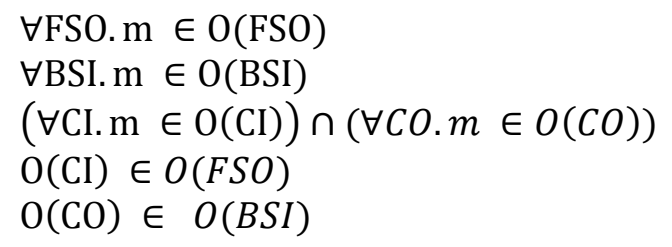

BSI.m : modelreference of input parameter of existing services will be located back of substituted services.

FSO.m : modelreference of output parameter of existing services will be located front of substituted service.

CI.m : modelreference of input parameter of candidate substitutable service.

CO.m : modelreference of output parameter of candidate substitutable service.

$O$ : ontology representing relationship service and servicêpgrameters.

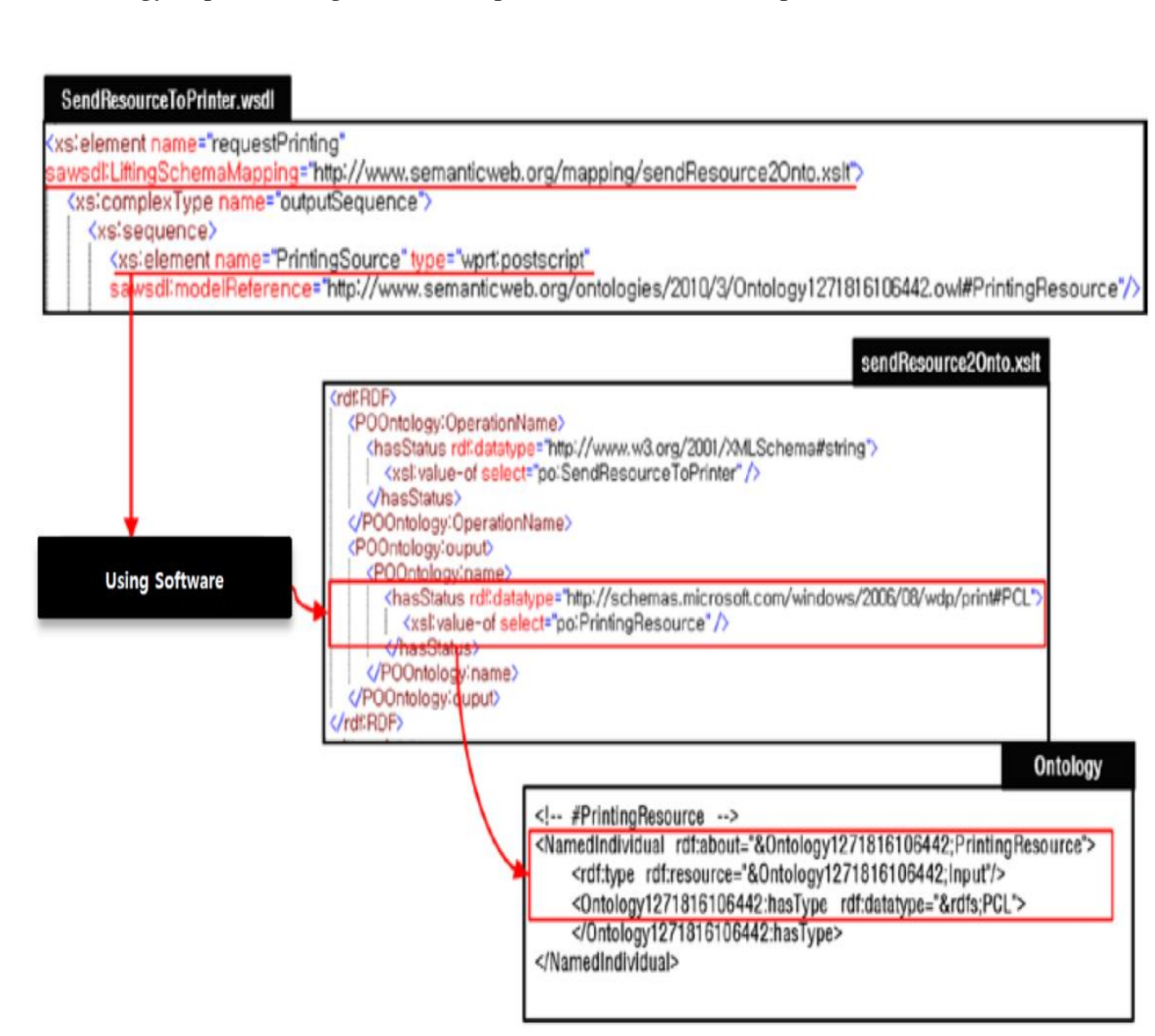

Figure 4. Lifting Schema Mapping Process 


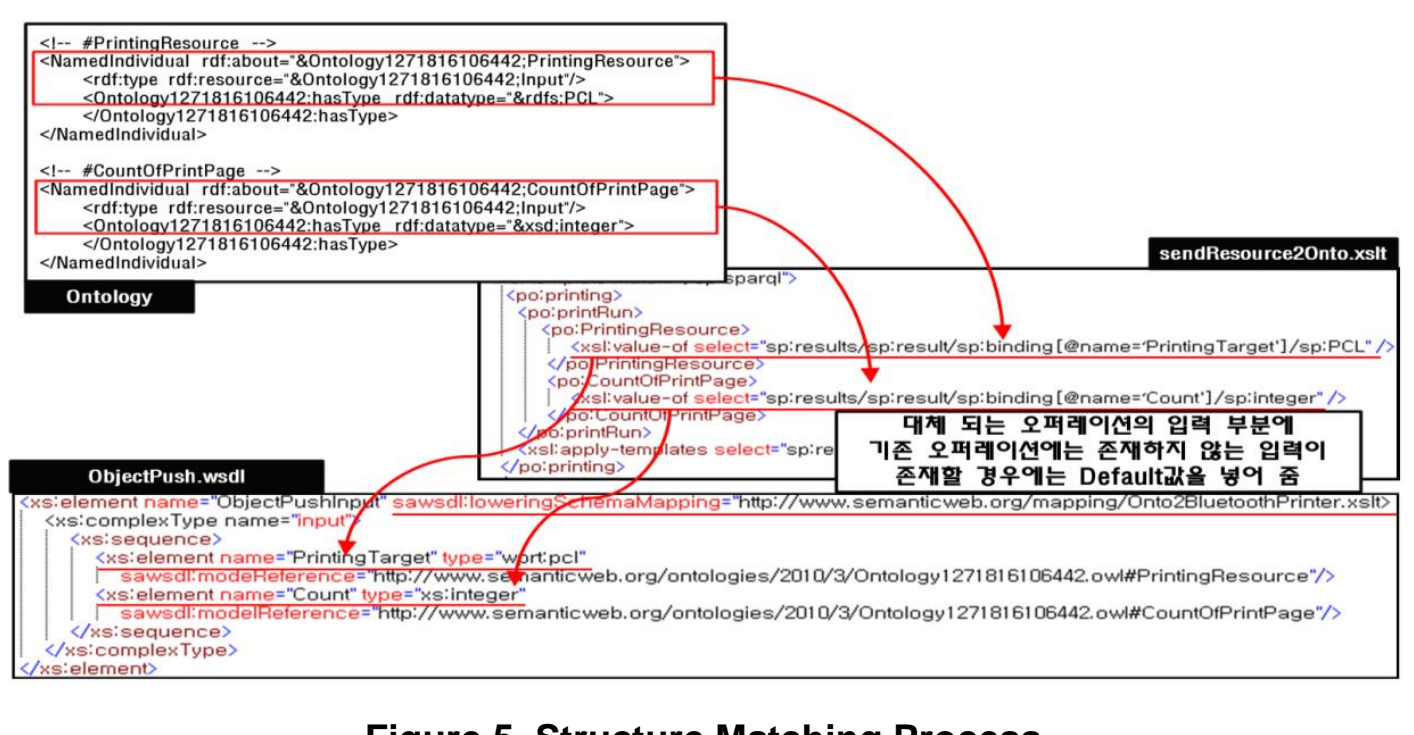

Figure 5. Structure Matching Process

According the location of services will connect to substityted service; candidate service satisfies equations from Eq. 1 to Eq. 4 or from Eq. 1 to Eq 3 and Eq. 5. The third and last process is lifting and lowering process between different parameter types. Figure 4 shows the parameter type matching process. The sendResource 20 nto.xslt can be used as a schema for mapping from SendResourceToPrinter.wsdl to concepts in Ontology. The type of output parameter in WSDL document is postscript, and the normal type of output parameter defined PCL in Ontology. We support sendResource2Onto.xslt to mapping between different parameter types.

This process is displayed in Figure 5. Assuming SendResourceToPrinter.wsdl in Figure 4 must connect to ObjectPush.wsdl ObjectPush.wsdl required the Count input is integer type and mandatory parameter. In this case, we support sendResource2Onto.xslt to allocate default value into Count input parameter automatically.

\subsection{Quality Criteria for Elementary Services}

In ubiquitous environment, we have to guarantee 'seamless' service to the user. To address this problem, we define the Quality-of-Services (QoS) as a set of perceivable characteristics expressed ip-user-friendly language with quantifiable parameters that may be subjective or objective The characteristics of quality and their parameters are based on the user or client requirements. In addition, we consider the where the service is. The user decides whether the location is important or not. Our system makes the candidate service list according the user decision. We consider four generic quality criteria for elementary services:

- execution time: the execution time of a operation is defined as the time spent by the system execution that operation, including the time spent execution run-time and the time spent lifting and lowering schema mapping. The execution time is computed using the expression

$$
q_{\text {time }}(o p)=T_{\text {process }}(o p)+T_{\text {mapping }}(\text { onto, } o p) \text {, }
$$

meaning that the execution time is the sum of the processing time $T_{\text {process }}(o p)$ and the schema mapping time $T_{\text {mapping }}($ onto, $o p$ ). The schema mapping time is estimated based on executions of lifting and lowering schema mapping, i.e., 


$$
T_{\text {mapping }}(\text { onto, } o p)=T_{\text {lifting }}(\text { onto, } o)+T_{\text {lowering }}(o, i) \text {, }
$$

where $T_{\text {lifting }}$ (onto, $o$ ) is lifting schema mapping time between operation group ontology onto and operation output parameter $o$, and $T_{\text {lifting }}($ onto, $o$ ) is lowering schema mapping time between operation onto and operation input parameter $i$.

- execution price: Given an operation $o p$ of service, the execution price $q_{p r}(o p)$ is the fee that an operation requester has to pay for invoking the operation $F_{\text {invoke }}(o p)$. In addition, it includes the fee to use software $F_{\text {invoke }}($ soft $)$ which supports schema mapping.

$$
q_{p r}(o p)=F_{\text {invoke }}(o p)+F_{\text {invoke }}(\text { soft })
$$

- successful execution rate: The successful execution rate $q_{\text {rat }}(o p)$ of an operation $o p$ is that how many the operation is completed without failed or error occurred. The value of the success rate is computed from data of past invocations using the expression

$$
q_{\text {rat }}(\text { op })=N_{\text {completed }}(\text { op }) / T, \quad /(9)
$$

where $N_{\text {completed }}(o p)$ is the number of times that the operation op has been successfully completed, and $T$ is the total number of invocations. $P$

- use frequency: Given an operation op of service, the use frequency $q_{f r}(o p)$ is that how many the operation is invoked $N_{\text {selection }(0 p)}$ ) by the operation requester. The expression is

$$
q_{f r}(\text { op })=\sum_{1}^{n}\left(N_{i} \text { (onto }\right) / N_{\text {serection }}(o p \text {, onto }) \text {, }
$$

where $\sum_{1}^{n} N_{i}$ (onto) is the total number of invoked operation in same operation group ontology onto.

Our system makes the recommend operations list used by the weight of user requirement. The users are able to decide that how much each qos criteria are important. Our system supports ranked operation list that is the result to calculate with weighted qos criteria. The ranked operation list is made (by) the expression

$$
\mathrm{Q}=\left(\mathrm{q}_{\text {time }}(\mathrm{op}) \times \mathrm{W}_{\mathrm{t} \text { me }}(\mathrm{op})\right)+\left(\mathrm{q}_{\mathrm{pr}}(\mathrm{op}) \times \mathrm{W}_{\mathrm{pr}}(\mathrm{op})\right)+\left(\mathrm{q}_{\mathrm{rat}}(\mathrm{op}) \times \mathrm{W}_{\text {rat }}(\mathrm{op})\right)+
$$

where $W_{\text {time }}(o p)$ is the weight of execution time that is the value entered by user. For examp e, there are two candidate operations (Table 1). The candidate operation B is free, many users used this operation. The candidate operation $\mathrm{C}$ is faster than candidate operation B, but user has to pay using this operation. The user, who needs seamless services now, prefers a good software performance.

Table 1. Example of Candidate Operations

\begin{tabular}{lccc}
\hline \multicolumn{1}{c}{ QoS criteria } & Candidate operation B & Candidate operation C & Weight \\
\hline Execution time & 0.15 & 0.5 & 4 \\
Execution price & 0 & 0.4 & 1 \\
Successful execution rate & 0.9 & 0.9 & 0 \\
Use frequency & 0.4 & 0.2 & 1 \\
\hline
\end{tabular}


Table 2 shows the calculated result according to expression (11). Although many users had used the operation B, operation C is fit to current user, who wants software which supports good performance.

Table 2. The Result of $Q$

\begin{tabular}{lcc} 
& Candidate operation B & Candidate operation C \\
\hline$\left(q_{\text {time }}(o p) \times W_{\text {time }}(o p)\right)$ & 0.6 & 2.0 \\
$\left(q_{p r}(o p) \times W_{p r}(o p)\right)$ & 0 & 0.4 \\
$\left(q_{r a t}(o p) \times W_{\text {rat }}(o p)\right)$ & 0 & 0 \\
$\left(q_{f r}(o p) \times W_{f r}(o p)\right)$ & 0.4 & 1.0
\end{tabular}

Operation of the T-Score calculated quality replacement can be found for prioritized operations to compare the operation method. Operation of the particular quality T-Score calculated to determine the ranking of the guality of the operation can be tailored to complement the problems.

For example, operation $\mathrm{A}$ is the faster than operation B. This means that operation $\mathrm{B}$ requires the time of performing replacement more than operation $\mathrm{B}$, but operation $\mathrm{A}$ uses more than 1000 times lower than a $\mathrm{B}$ operation th the user determines the first priority is operation replacement time and the next priority is operation using counts, the operation $\mathrm{A}$ is best choice better than operation B. However, in) user's perspective, the operation B is a good operation, because operation $B$ has similar replacement time of operation A and it has much higher using time than operation A. Phus, the quality of a particular ranking the recommended build for this operation to exclude situations where the quality of this study were compared to previous operations to solve problems in standard scores (Standard score) [13] method of calculating T-Score was used.

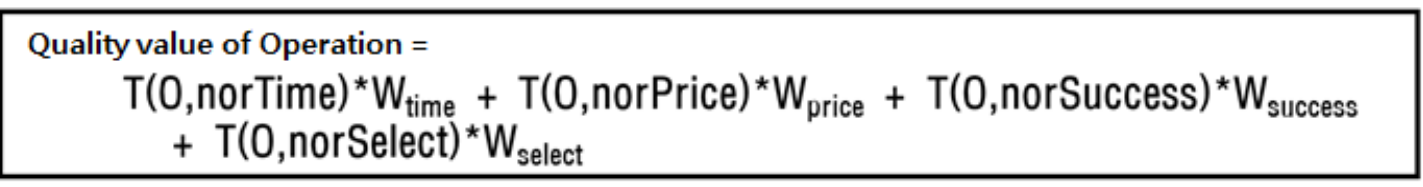

Figure 6. Final Value of Quality about Operation

$$
\begin{aligned}
& T(01, \text { time })=\left[\frac{01 \cdot \text { norTime-m }}{S}\right] \times 10+50 \\
& S=\sqrt{\frac{\left(01 . \text { norTime-m) }{ }^{2}+(02 \cdot \text { norTime-m) })^{2}+\cdots+(\text { On.norTime }-m)^{2}\right.}{n}} \\
& \mathrm{~m} \text { : average, } \mathrm{n} \text { : number of operation }
\end{aligned}
$$

Figure 7. T-Score Calculation 


\section{Conclusion}

When ubiquitous environment occur the mobility of heterogeneous services, some services have to be replaced to another service. Therefore, the user spends a lot of time to find the appropriate service. To provide 'seamless' service to users, many studies were used to substitute the service. However they do not consider the characteristic of ubiquitous computing environment. Furthermore, they do not support ordering substitution list for fit of the user's requirements.

In this paper, to solve these limitations, we support the substitution operation method based on SAWSDL mechanism. In addition, we define the quality factors of operation to find the operation, and our system uses the weight value from user. It can disdover operation which is more suitable for user requirements.

Future research directions of this study are as follows. Currently, our system can support the selection of the operation has specific position, but it needs the location filtering process. This filtering process includes location information on street. The using of space and distance information is expected to be selected more substitutableoperation.

\section{Acknowledgements}

This research was supported by the Global resource utilization project in science and technology from operation of KOSEN of the National Research Foundation (NRF) funded by the Ministry of Science, ICT \& Future Planing(N-14-NM-IR09).

This paper is a revised and expanded version of a paper entitled [The Method for Service Recommendation in Ubiquitous Computing] presepted at [UCMA, Batam in Indonesia, Jun $18 \sim 21,2014]$.

\section{References}

[1] A. A. Abbasi and M Hursain, "A Qos Enhancement Framework for Ubiquitous Network Environments", IJAST, vol. 43, (2012),pp. 37-48.

[2] A. A. Abbas and A. Florentipa, "An Analysis of QoS specific Coherence Issues in Distributed Networks", IJAST, vol. 49, (2012), pp. 73-82)

[3] B. S. Jun, "SOA, What \& How, WowBooks, (2008).

[4] C. Brodersen, S. Bødkervand C. Nylandsted Klokmose, "Ubiquitous Substitution", Human Computer Interaction 07, (2007), pp. 179-192.

[5] D. Chappel, "Introducing SCA", In Proceeding of the CHAPPELL \& ASSOCIATES, (2007).

[6] G. Zayaraz and P. Kalamegam, "A test framework based on CPN model for functional testing of Web Service Composition TAST, vol. 53, (2013).

[7] H. J. Yin, Y.' Y. Hwang and K. C. Lee, "Task-based Services Composition for Ubiquitous Computing", Socia Com2011, (2011).

[8] Leng and B. Benatallah, "QoS-Aware Middleware for Web Services Composition", IEEE Transaction on Softyare engineering, vol. 30, (2004), pp. 311-327.

[9] N. Ibrahim, F. Le Mouel and Frenot, "Semantic Service Substitution in Pervasive Environments", International Journal of Services/Economics and Management,(2010).

[10] OASIS, “Web Services Quality Factors v1.0,” OASIS Working Draft, (2010) June 7.

[11] S. H. Oh, S. D. Kim and S. Y. Ryu, "UCQM: A Quality Model for Practical Evaluation of Ubiquitous Computing Systems", Journal of KIISE vol. 34, no. 4, (2007), pp. 342-358.

[12] Software Engineering-Product Quality-part 2: Internal Metrics. ISO/IEC TR 9126-2, (2003).

[13] Wikipedia, "Standard score", http://en.wikipedia.org/wiki/Standard_score.

[14] Wikipedia, "Quality of Service", http://en.wikipedia.org/ wiki/Quality_of_service

[15] J. Farrel and H. Lausen, "Semantic Annotations for WSDL and XML Schema", W3C Recommendation, 2007.08 (2007).

[16] X. Wang, T. Vitvar, M. Kerrigan and I.Toma. "A QoS aware selection model for semantic web services," In 4th International Conference on Service Oriented Computing (ICSOC 2006), pp. 390-401, (2006). 
[17] Y. Taher, D. Benslimane, M. C. Fauvet and Z. Maamar, "Towards an Approach for Web Services Substitution", IEEE IDEAS06, (2006), pp. 166-173.

\section{Authors}

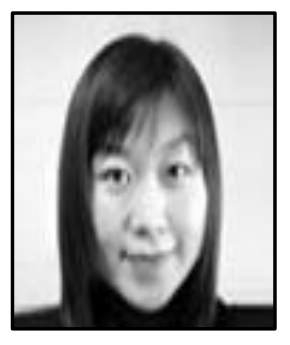

Yun-Young Hwang, She received B.E., M.E., and Ph.D. degrees in computer engineering from Chungnam National University in 2002, 2004, and 2011, respectively. In 2012 she joined KISTI (Korean Institute of Science and Technology Information), Korea where she is currently a researcher in Dept. of Overseas Information.

Her current areas include Big-Data Processing, Cloud-Computing, Ubiquitous Computing and Long-Term Preservation of Electronic Records. She has published over 30 technical articles in várious journals and conferences.

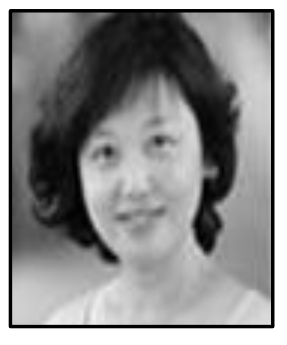

Jungsun Yoon, She receved B.S., N.S. degrees in computer science from Korea Advanced Institute of Science and Technology in 1991, and 1993 respectively. From 1993 to 2000 she worked for KRISS(Korea Research Institute of Standards and Science) as a senior researcher. In 2000 she joined KISTI(Korean Institute of Science and Technology Information), where she is currently a principal resêareher in Dept of Overseas Information.

Her current areas of interest include information service, usability and $\mathrm{HCI}$.

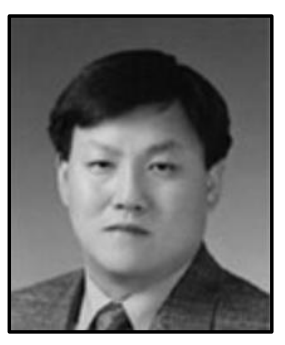

Kyu-Chul Lede, He received B.E., M.E., and Ph.D. degrees in Computer Engineering from Seoul National University in 1984, 1986, and 1996, respectively. In 1994 he worked as a visiting researcher at the IBM Almaden Research Center, San Jose, California. From 1995 to 1996, he worked as a Visiting Professor at the CASE Center at Syracuse University, Syracuse, New York. He is currently a Professor in the Department of Computer Engineering at Chungnam National University, Daejeon, Korea.

His current areas include Multimedia Database System, Hypermedia Systems, Object-Oriented Systems, and Digital Libraries. He has published over 100 technical articles in various journals and conferences. He is a member of ACM, IEEE Computer Society, and Korea Information Science Society. 
International Journal of Multimedia and Ubiquitous Engineering Vol. 9, No. 10 (2014)

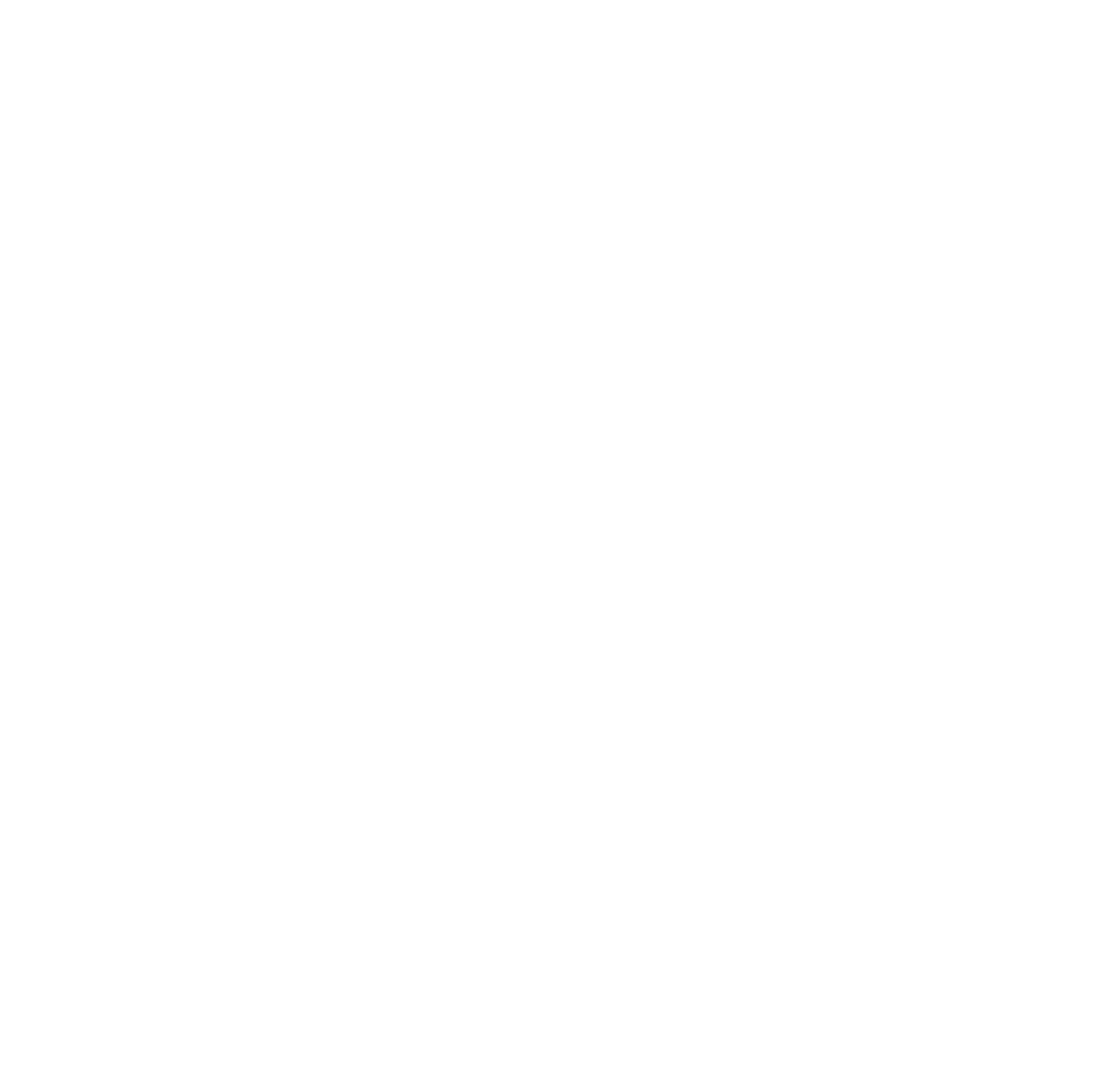

